\title{
Modelagem Matemática com o Software Mathema- tica na Simulação Computacional de um Secador de Leito Fluidizado em Regime Estacionário
}

Lourenço, J. R.

Escola Politécnica de Pernambuco

Universidade de Pernambuco

50.720-001 - Recife, Brasil

jobson.lourenço@gmail.com
Galdino, S. M. L.

Escola Politécnica de Pernambuco

Universidade de Pernambuco

50.720-001 - Recife, Brasil
Silva, J. D.

Escola Politécnica de Pernambuco

Universidade de Pernambuco

50.720-001 - Recife, Brasil

Resumo A modelagem matemática de sistemas fisicos, bem como o tratamento dado às equações ou sistemas de equações diferenciais aplicadas modelo, podem atuar de forma significativa no resultado encontrado. $O$ modelo gerado deve receber um tratamento numérico computacional, processo que pode tornar-se trabalhoso, na medida que o modelo seja mais complexo, devido principalmente às etapas pré-computacionais de discretização e padronização do sistema ao método a ser empregado. Nesse aspecto, pretende-se neste trabalho mostrar a aplicação do software Mathematica como um meio de dinamizar a modelagem e simulação de sistemas físicos, uma vez que este fornece alternativas automáticas de soluções de equações diferenciais de vários tipos, o que permite ao modelador focar no sistema modelado e não em técnicas e métodos numéricos.

\footnotetext{
Abstract Mathematical modeling of physical systems, as well as treatment of equations or set of differential equations applied to the model, can act significantly in the results found. The generated model should be treated numeric computational method, a process which can become laborious, as the model becomes more complex, mainly due pre-compute steps. In this way, this work aims to show application of software Mathematica as a mean to streamline the modeling and simulation of physical systems, since this eliminates such steps.
} 


\section{Introdução}

$\mathrm{Na}$ formulação de muitos modelos matemáticos, sistemas de equações diferencias são utilizados para representar o fenômeno físico. Contudo, o tratamento e solução de tais sistemas nem sempre é simples, sendo requeridas ferramentas computacionais eficazes para solução do modelo. Uma dessas ferramentas é o software Mathematica.

O Mathematica é um poderoso ambiente de programação matemática, dispondo de ferramentas numéricas, simbólicas e gráficas, de modo a auxiliar em cada um dos aspectos da resolução de problemas matemáticos. Este software tem sido amplamente empregado na investigação e análise de problemas em engenharia, matemática e física, bem como em economia e outras ciências, e também pode ser usado como uma linguagem de programação de alto nível. Além disso, o Mathematica.possui uma interface simples e intuitiva, facilitando o trabalho do usuário [1].

Neste aspecto, o Mathematica pode ser utilizado na solução de problemas de modelagem através da função intrínseca NDSolve, que é, de forma geral, um solucionador numéricos de equações diferenciais. Ele pode lidar com uma ampla gama de equações diferenciais ordinárias (EDOs), bem como equações diferenciais parciais (EDPs). NDSolve também pode resolver algumas equações algébrico-diferenciais (EADs), que normalmente são uma mistura de equações diferenciais e algébricas [2].

Como forma de exemplificar essa ferramenta, neste trabalho pretende-se mostrar o uso do NDSolve para a solução de um problema de modelagem e simulação matemática de um secador de leito fluidizado bifásico em regime estacionário [3]. O artigo divide-se da seguinte forma: a Seção 2 descreve em alguns detalhes o NDSolve, a Seção 3 descreve o modelo físico do secador de Leito Fluidizado, a Seção 4 apresenta resultados e discussões e a Seção 5 as conclusões.

\section{A função NDSolve}

Nesta seção a função intrínseca do Mathematica, será descrita em alguns detalhes [3]. A função NDSolve encontra a solução numérica de equações diferenciais, bem como sistemas de equações diferenciais acopladas, conseguindo resolver vários tipos de EDOs e EDPs, além de manusear algumas equações algébrico-diferenciais (EADs) que consistem de um problema que envolve equações diferencias e algébricas.

Sintaxe:
- $\quad$ DSolve $[\{$ eqn 1, eqn $2, \ldots, y,\{x, x \operatorname{mín}, x m a ́ x\}]$, encontra uma solução numérica para a função $y$, com $x$ no intervalo [xmín,xmáx].

- $\quad$ DS Solve $\left[\left\{\right.\right.$ eqn1,eqn $2, \ldots, \quad y_{1}, y_{2}, \ldots, y_{n}$, $\left.\left.x, x_{m i ́ n}, x_{m a ́ x}\right\}\right]$, encontra uma solução numérica para as funções $y i$, com $x$ no intervalo $\left[x_{m i ́ n}, x_{m a ́ x}\right]$.

NDSolve apresenta a solução para as funções yi como funções interpoladas (InterpolatingFunction), as quais fornecem aproximações para $y i$ ao longo do intervalo de valores $x \min$ até $x \max$ para a variável independente $x$. Para uma visualização da resposta pode-se traçar o gráfico da função obtida no respectivo intervalo por meio do seguinte comando:

$$
\begin{gathered}
\text { Plot }\left[\text { Evaluate }\left[y_{i}[x] / . \text { sol }\right],\left\{x, x_{\min }, x_{\operatorname{má} x}\right\},\right. \\
\text { PlotRange } \rightarrow \text { All }]
\end{gathered}
$$

Nota-se ainda que quando se usa o NDSolve as condições iniciais e/ou de contorno dadas devem ser suficientes para determinar a solução para yi completamente, uma vez que este comando encontrará uma solução numérica, - diferentemente do comando DSolve que encontra, se possível uma solução algébrica, podendo esta conter graus de liberdade adicionais. Em um caso típico, se existir uma equação diferencial com a n-ésima derivada, então é necessário fornecer ao software condições iniciais para as derivadas até grau $(n-1)$, ou condições de contorno em $n$ pontos.

$\mathrm{Na}$ maioria dos casos, todas as condições iniciais fornecidas envolvem algum valor de $x$, digamos $x_{0}$. Como resultado, deve-se fornecer explicitamente os extremos do intervalo de $x, x_{\text {mín e }} x_{m a ́ x}$. Caso o intervalo seja especificado como $\left\{x, x_{1}\right\}$, o Mathematica irá automaticamente gerar uma solução sobre o intervalo $\left[x_{0}, x_{1}\right]$.

O NDSolve permite ainda outras opçõe, mostradas na Tabela 1.

Tabela 1. Opções para a função NDSolve.

\begin{tabular}{l|l|l} 
Nome & $\begin{array}{l}\text { Valor } \\
\text { padrão }\end{array}$ & Descrição \\
\hline \hline MaxSteps & Automatic & $\begin{array}{l}\mathrm{N}^{0} \text { máximo de } \\
\text { passos para } x\end{array}$ \\
\hline MaxStepFraction & $1 / 10$ & $\begin{array}{l}\text { Fração } \\
\text { máxima do }\end{array}$ \\
\hline
\end{tabular}




\begin{tabular}{|c|c|c|}
\hline & & $\begin{array}{l}\text { intervalo } \\
\text { coberta } \\
\text { cada passo }\end{array}$ \\
\hline StartingStepsSize & Automatic & $\begin{array}{l}\text { Tamanho } \\
\text { inicial } \\
\text { passo de } x\end{array}$ \\
\hline MaxStepsSize & Automatic & $\begin{array}{l}\text { Tamanho } \\
\text { máximo do } \\
\text { passo usado } \\
\text { para } x\end{array}$ \\
\hline NormFunction & Automatic & $\begin{array}{lr}\text { norma usada } \\
\text { para } \\
\text { estimativa do } \\
\text { erro }\end{array}$ \\
\hline AccuracyGoal & Automatic & $\begin{array}{ll}\text { Digitos de } & \text { de } \\
\text { exatidão } & \\
\text { absoluta } & \\
\text { solicitados } & \end{array}$ \\
\hline PrecisionGoal & Automatic & $\begin{array}{l}\text { Digitos de } \\
\text { precisão } \\
\text { solicitados }\end{array}$ \\
\hline DependentVariables & & $\begin{array}{l}\text { Lista de todas } \\
\text { as variáveis } \\
\text { dependentes }\end{array}$ \\
\hline EvaluationMonitor & None & $\begin{array}{l}\text { Expressão que } \\
\text { avalia sempre } \\
\text { que a função é } \\
\text { evoluida }\end{array}$ \\
\hline InterpolationOrder & Automatic & $\begin{array}{l}\text { O grau de } \\
\text { interpolação } \\
\text { da resposta }\end{array}$ \\
\hline StepMonitor & None & $\begin{array}{l}\text { Expressão que } \\
\text { avalia quando } \\
\text { um passo é } \\
\text { feito }\end{array}$ \\
\hline WorkingPrecision & $\begin{array}{l}\text { MachinePre } \\
\text { cision }\end{array}$ & $\begin{array}{l}\text { Precisão usada } \\
\text { nas operações } \\
\text { internas }\end{array}$ \\
\hline Method & Automatic & Método usado \\
\hline
\end{tabular}

Nota-se que:

i. NDSolve adapta o tamanho do passo para que o erro estimado na solução fique dentro das tolerâncias especificadas por PrecisionGoal e AccuracyGoal.

ii. A opção NormFunction $\rightarrow f$ especifica que o erro estimado para cada um dos $y_{i}$ deve ser uma ser uma combinação, usando $f[\{e 1, e 2, \ldots\}]$.

iii. AccuracyGoal efetivamente especifica o valor do erro absoluto local permitido em cada passo na busca de uma solução, enquanto PrecisionGoal especifica o erro relativo local.

iv. Se as soluções necessitam de precisão quando seus valores estão perto de zero, AccuracyGoal deve ser ajustado maior, ou até Infinity. v. A configuração padrão de Automatic para AccuracyGoal e PrecisionGoal é equivalente a WorkingPrecision / 2.

vi. O ajuste para MaxStepFraction especifica o passo máximo a ser feito pelo NDSolve como uma fração do intervalo de valores da variável independente.

vii. Com DependentVariables $\rightarrow$ Automatic, NDSolve tenta determinar as variáveis dependentes através da análise das equações dadas.

viii. As definições possíveis explicitadas para a opção Method incluem, mas não limitam-se a: Adams, $B D F$, ExplicitRungeKutta, ImplicitRungeKutta, SympleticPartitonedRungeKutta.

ix. A configuração InterpolationOrder $\rightarrow$ All, especifica que NDSolve deve gerar soluções que utilizam interpolação da mesma ordem que o método subjacente utilizado.

Por fim, ressalta-se que para fazer uso de todas as opções implementadas no NDSolve, anteriormente citadas deve-se inicialmente preparar o ambiente de programação do Mathematica, por meio dos seguintes comandos:

Needs["DifferentialEquations`NDSolveProblems " ]; Needs["DifferentialEquations`NDSolveUtilities '"];

\section{Modelo Matemático do Secador de Leito Fluidizado}

Nessa seção será descrito o modelo físico do secador de leito fluidizado que foi utilizado como estudo de caso para a utilização do Mathematica.

O processo de secagem de combustíveis sólidos utilizando secadores de leito fluidizado é amplamente utilizado na indústria devido a sua eficiência graças ao grande contato entre sólido e gás, resultando em alta transferência de calor para secagem. Do ponto de vista termodinâmico, o processo de secagem de combustíveis utilizando-se um secador de leito fluidizado envolve os fenômenos de acumulação, dispersão e transferência de calor, podendo ser modelado a partir das equações de transferência de momento, energia e massa que resultam em um sistema de equações diferencias parciais de segunda ordem [4].

Um secador de leito fluidizado pode ser modelado a partir do balanço de energia e de massa [3]. As equações 
de balanço de energia e massa para as fases gasosa e sólida foram simplificadas com as seguintes hipóteses simplificadoras para obter um modelo simplificado para o secador de leito fluidizado em regime estacionário:

i. modelo unidimensional;

ii. sistema de secagem não isotérmico,

iii. as temperaturas das fases gasosa e sólida são modeladas através de modelos de dispersão térmica e

iv. os balanços de massa para umidade das fases gasosa e sólida são modelados com os efeitos de dispersão axial

v. os termos de acumulação para os balanços de energia e massa são desprezados.

Baseado nessas hipóteses, o modelo matemático simplificado para o secador de leito fluidizado em regime estacionário é formado pelas equações simplificadas de transporte energia e massa que descrevem o comportamento das temperaturas e umidades no secador. Assim, as equações simplificadas que formam o modelo são expressas por:

Balanço de energia para a fase gasosa:

$$
\begin{aligned}
& \varepsilon_{f g} K_{f g, e f f} \frac{d^{2} T_{f g}}{d z^{2}}+\frac{\varepsilon_{f g} C_{P, f g} \rho_{f g} Q_{f g}}{A_{s}} \frac{d T_{f g}}{d z}- \\
& (h a)_{f g s}\left(T_{f g}-T_{s}\right)-\frac{C_{v a}(h a)_{f g s}}{Q_{f g}}\left(Y_{s}-Y_{f g}\right)=0
\end{aligned}
$$

Condições de contorno:

$$
\begin{aligned}
& \left.K_{f g, e f f} \frac{d T_{f g}}{d z}\right|_{z=0^{+}}=\frac{C_{P, f g} \rho_{f g} Q_{f g}}{A_{s}}\left(\left.T_{f g}\right|_{z=0^{+}}-T_{f g, 0}\right) \\
& \left.\frac{d T_{f g}}{d z}\right|_{z=L}=0
\end{aligned}
$$

Balanço de energia para a fase sólida:

$$
\begin{aligned}
& \varepsilon_{s} K_{s, e f f} \frac{d^{2} T_{s}}{d z^{2}}-\frac{\varepsilon_{s} C_{P, s} G_{t, s}}{A_{s}} \frac{d T_{s}}{d z}+(h a)_{f g s}\left(T_{s}-T_{f g}\right) \\
& +\lambda \frac{C_{v a}(h a)_{f g s}}{Q_{g}}\left(Y_{s}-Y_{f g}\right)=0
\end{aligned}
$$

Condições de contorno:
$\left.K_{s, e f f} \frac{d T_{s}}{d z}\right|_{z=0^{+}}=\frac{C_{P, s} G_{t, s}}{A_{s} \rho_{s}}\left(\left.T_{s}\right|_{z=0^{+}}-T_{s, 0}\right)$

$\left.\frac{d T_{s}}{d z}\right|_{z=L}=0$

Balanço de massa da fração molar para a fase sólida:

$$
\varepsilon_{s} D_{s} \frac{d^{2} Y_{s}}{d z^{2}}-\frac{\varepsilon_{s} G_{t, s}}{A_{s}} \frac{d Y_{s}}{d z}-(k a)_{f g s}\left(Y_{s}-Y_{f g}\right)=0
$$

Condições de contorno:

$$
\begin{aligned}
& \left.\frac{d T_{s}}{d z}\right|_{z=0^{+}}=\frac{G_{t, s}}{A_{s} \varepsilon_{s} \rho_{s} D_{s}}\left(\left.T_{s}\right|_{z=0^{+}}-T_{s, 0}\right) \\
& \left.\frac{d T_{s}}{d z}\right|_{z=L}=0
\end{aligned}
$$

Quando as equações são adimensionalizadas elas podem ser expressas por:

Balanço de energia para a fase gasosa, adimensionalizada:

$$
\frac{d^{2} \theta_{f g}}{d \mu^{2}}-\alpha_{1} \frac{d \theta_{f g}}{d \mu}-\alpha_{2}\left(\theta_{f g}-\theta_{s}\right)-\alpha_{3}\left(Y_{s}-Y_{f g}\right)=0
$$

Condições de contorno adimensionalizadas para a fase gasosa:

$$
\begin{aligned}
& \left.\frac{d \theta_{f g}}{d \mu}\right|_{\mu=0^{+}}=-\alpha_{4}\left(\left.\theta_{f g}\right|_{=0^{+}}-1\right) \\
& \left.\frac{d \theta_{f g}}{d \mu}\right|_{\mu=1}=0
\end{aligned}
$$

Balanço de massa para a fase sólida, adimensionalizada:

$$
\frac{d^{2} Y_{s}}{d \mu^{2}}-\beta_{5} \frac{d Y_{s}}{d \mu}-\beta_{6}\left(Y_{s}-Y_{f g}\right)=0
$$

Condições de contorno adimensionalizadas para a fase sólida: 


$$
\begin{aligned}
& \left.\frac{d \theta_{s}}{d \mu}\right|_{\mu=0^{+}}=\beta_{4}\left(\left.\theta_{s}\right|_{=0^{+}}-1\right) \\
& \left.\frac{d \theta_{s}}{d \mu}\right|_{\mu=1}=0
\end{aligned}
$$

Balanço de energia para a fase sólida, adimensionalizada:

$$
\frac{d^{2} \theta_{s}}{d \mu^{2}}-\beta_{1} \frac{d \theta_{s}}{d \mu}-\beta_{2}\left(\theta_{s}-\theta_{f g}\right)-\beta_{3}\left(Y_{s}-Y_{f g}\right)=0
$$

Condições de contorno adimensionalizadas para a fase sólida:

$$
\begin{aligned}
& \left.\frac{d \theta_{s}}{d \mu}\right|_{\mu=0^{+}}=\beta_{4}\left(\left.\theta_{s}\right|_{=0^{+}}-1\right) \\
& \left.\frac{d \theta_{s}}{d \mu}\right|_{\mu=1}=0
\end{aligned}
$$

Balanço de massa da fração molar para a fase sólida, adimensionalizada:

$$
\frac{d^{2} Y_{s}}{d \mu^{2}}-\beta_{5} \frac{d Y_{s}}{d \mu}-\beta_{6}\left(Y_{s}-Y_{f g}\right)=0
$$

Condições de contorno adimensionalizadas da fração molar para a fase sólida:

$$
\begin{aligned}
& \left.\frac{d Y_{s}}{d \mu}\right|_{\mu=0^{+}}=\beta_{7}\left(Y_{s}-Y_{s, 0}\right) \\
& \left.\frac{d Y_{s}}{d \mu}\right|_{\mu=1}=0
\end{aligned}
$$

Onde os parâmetros adimensionais da fase gasosa $\alpha_{\mathrm{i}}$ e os parâmetros adimensionais da fase sólida $\beta_{i}$ são dados por:
Tabela 2. Coeficientes adimensionais utilizados.

\begin{tabular}{c|c}
\hline $\begin{array}{c}\text { Coeficientes da fase } \\
\text { gasosa }\end{array}$ & $\begin{array}{c}\text { Coeficientes da fase } \\
\text { sólida }\end{array}$ \\
\hline$\alpha_{1}=\frac{C_{P, f g} \rho_{f g} Q_{f g} L}{A_{s} K_{f g, e f f}}$ & $\beta_{1}=\frac{C_{P, s} G_{t, s} L}{A_{s} K_{f g, e f f}}$ \\
$\alpha_{2}=\frac{(h a)_{f g s} L^{2}}{\varepsilon_{f g} K_{f g, e f f}}$ & $\beta_{2}=\frac{(h a)_{f g s} L^{2}}{\varepsilon_{s} K_{s, e f f}}$ \\
$\alpha_{3}=\frac{C_{v a}(h a)_{f g s} L^{2}}{\varepsilon_{f g} K_{f g, e f f} Q_{f g} T_{f g, 0}}$ & $\beta_{3}=\frac{\lambda C_{v a}(h a)_{f g s} L^{2}}{\varepsilon_{s} K_{s, e f f} Q_{f g} T_{s, 0}}$ \\
$\alpha_{4}=\frac{C_{P, f g} \rho_{f g} Q_{f g} L}{A_{s} K_{f g, e f f}}$ & $\beta_{4}=\frac{C_{P, s} G_{t, s} L}{A_{s} K_{s, e f f}}$ \\
& $\beta_{5}=\frac{G_{t, s} L}{A_{s} \rho_{s} D_{s}}$ \\
& $\beta_{6}=\frac{(k a)_{f g s} L^{2}}{\varepsilon_{s} D_{s}}$ \\
& $\beta_{7}=\frac{G_{t, s} L}{A_{s} \rho_{s} D_{s}}$ \\
\hline
\end{tabular}

\section{Resultados e Discussões}

O modelo matemático anteriormente descrito foi solucionado utilizando a função NDSolve do Mathematica. Como resultado tem-se o comportamento da temperatura da fase gasosa e da temperatura e fração molar da fase sólida (Tfg , Ts e Ys, respectivamente), em função da posição. Para o cálculo dos coeficientes adimensionais $\alpha i$ e $\beta i$ foram utilizados os seguintes parâmetros [5]:

Tabela 3. Parâmetros utilizados na simulação do modelo.

\begin{tabular}{|c|c|}
\hline$\varepsilon_{f g}=0,460$ & $\varepsilon_{s}=0,780$ \\
\hline$K_{f g}=1,94 \times 10^{-5}$ & $K_{s, e f f}=1,94 \times 10^{-5}$ \\
\hline$C_{p, f g}=624$ & $C_{p, s}=9,53 \times 10^{-2}$ \\
\hline$\rho_{f g}=0,921$ & $\rho_{s}=289$ \\
\hline$Q_{f g}=2,56$ & $G_{t, s}=4,62 \times 10^{-2}$ \\
\hline$Y_{f g, 0}=0,500$ & $Y_{f g, 0}=0,500$ \\
\hline$T_{f g, 0}=220$ & $T_{f g, 0}=90$ \\
\hline$(k a)_{f g s}=3,23 \times 10^{-2}$ & $A_{s}=6,789$ \\
\hline$(h a)_{f g s}=0,216$ & $D_{s}=0,326$ \\
\hline$q_{t}=51,6$ & $\lambda=540$ \\
\hline
\end{tabular}

Com o resultado obtido, por meio de funções interpoladas (InterpolatingFunction), foi traçado os gráficos apresentados nas Figuras 1, 2 e 3. 


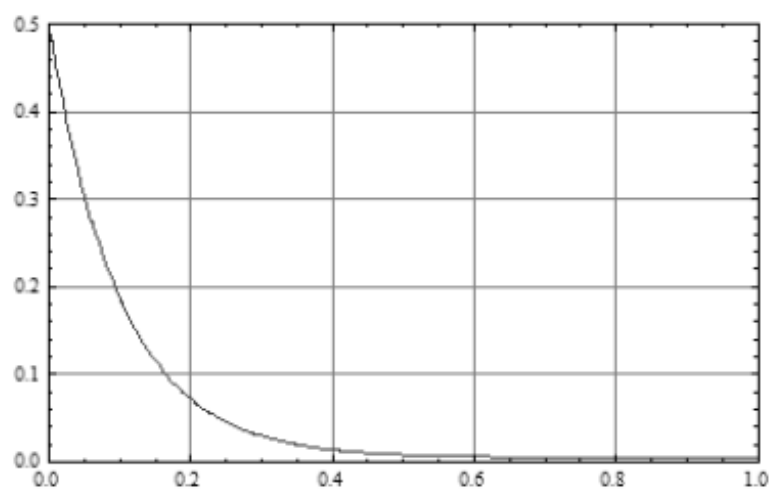

Fig 1. Perfil da Fração molar da fase sólida em função da posição obtido com o Software Mathematica

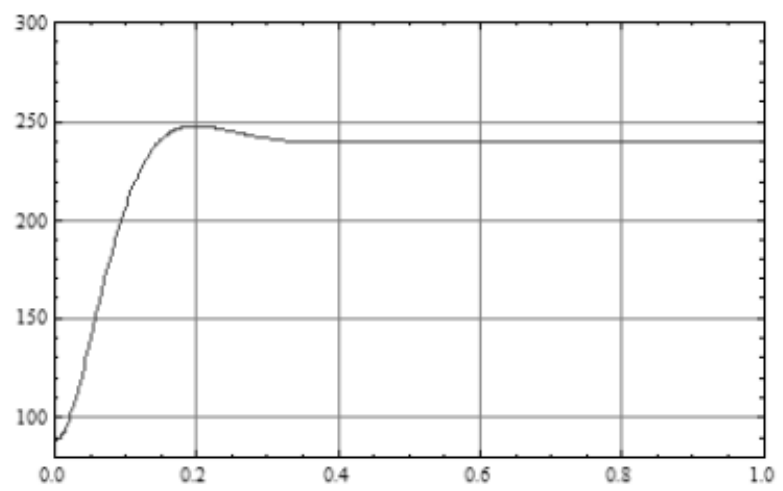

Fig 2. Perfil da temperatura da fase sólida em função da posição obtido com o Software Mathematica.

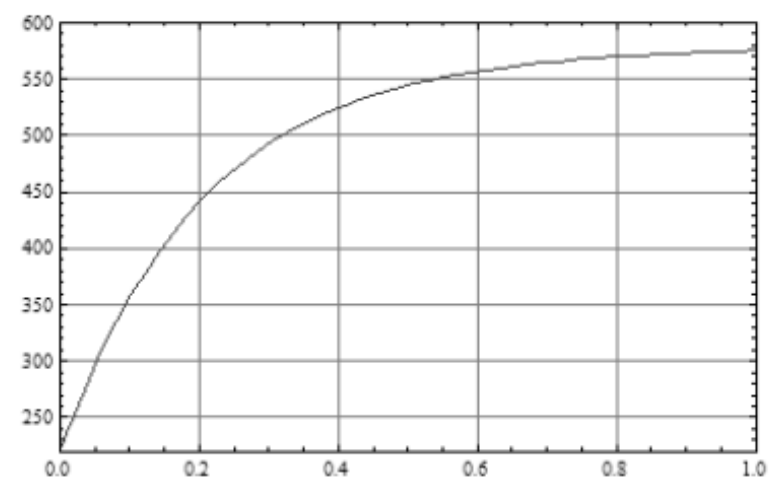

Fig 3. Perfil da temperatura da fase gasosa em função da posição obtido com o Software Mathematica

A Figura 1 mostra o perfil da fração molar da fase sólida em função da posição. Nota-se aqui que a fração molar da fase sólida torna-se praticamente constante a partir de $z \cong$ 0,5 . Tal fato ocorre devido ao equilíbrio do fluxo de calor no secador de leito fluidizado. Nota-se ainda que $Y_{s} \approx 0$, mostrando que a água foi eliminada do sólido [3].
A Figura 2 mostra o perfil de temperatura da fase sólida em função da posição. Nota-se por inspeção que a temperatura se mantém constante a partir de $z \cong 0,35 \mathrm{~m}$, devido ao equilíbrio do fluxo de calor entre as fases sólida e gasosa dentro do secador [3].

A Figura 3 mostra o perfil de temperatura da fase gasosa em função da posição. Nota-se que a temperatura tende a estabilizar-se a partir de $z \cong 0,8 \mathrm{~m}$. Esse comportamento, com um tempo de acomodação maior é verificado devido ao aumento da agitação molecular produzido pelo ar quente ao longo do secador [3].

\section{Conclusão}

Diante do cenário energético atual, a busca de meios renováveis e mais eficientes para geração de energia é de importância estratégica. Dessa forma buscar novos meios de estudar os processos de geração de energia, por meio de simulação computacional utilizando-se ferramentas avançadas, pode dinamizar o processo de análise, com ganho de produtividade nos resultados obtidos na modelagem.

Nesse aspecto, salienta-se como contribuição deste trabalho a aplicação do software o Mathematica como um meio de dinamizar a modelagem e simulação de sistemas térmicos uma vez que este mostra-se eficiente, robusto e versátil na resolução de vários tipos de modelos matemáticos, tornando sua utilização muito abrangente. Contudo, ressalta-se que embora o Mathematica tenha eliminado as etapas preliminares de discretização e padronização do modelo para a utilização do método, o conhecimento de qual método empregar, bem como dos ajustes que devem ser feitos para a obtenção da solução desejada tornam-se cruciais ao se utilizar este software.

Assim, a utilização do Mathematica para solução de problemas de modelagem e simulação representa um campo promissor a ser explorado em futuros trabalhos.

\section{Notações}

Kfg Condutividade térmica da fase gasosa, $\mathrm{kcal} / \mathrm{ms}^{\circ} \mathrm{C}$

Tfg Temperatura da fase gasosa, ${ }^{\circ} \mathrm{C}$

z Variável espacial, $\mathrm{m}$

Cp,fg Capacidade calorífica da fase gasosa, $\mathrm{kcal} / \mathrm{kg}^{\circ} \mathrm{C}$

Qfg Vazão da fase gasosa, $\mathrm{m}^{3} / \mathrm{s}$

As Área da seção transversal, $\mathrm{m}^{2}$ 
(ha)fgs Coeficiente de transferência de calor gássólido, $\mathrm{kcal} / \mathrm{m}^{3} \mathrm{~s}^{\circ} \mathrm{C}$

Ts Temperatura na fase sólida, ${ }^{\circ} \mathrm{C}$

qt Fluxo de calor, kcal/s

Ys Fração molar da fase sólida, mol/mol

$\mathrm{Ks}$,eff Condutividade térmica da fase sólida, $\mathrm{kcal} / \mathrm{ms}^{\circ} \mathrm{C}$

$\mathrm{Cp}, \mathrm{s} \quad$ Capacidade calorífica da fase sólida, $\mathrm{kcal} / \mathrm{kg}^{\circ} \mathrm{C}$

Gt,s Vazão de sólido, $\mathrm{kg} / \mathrm{s}$

(ka)fgs Coeficiente de transferência de massa gás-sólido, $\mathrm{kg} / \mathrm{m}^{3} \mathrm{~s}$

Ds Difusão no sólido, $\mathrm{m}^{2} / \mathrm{s}$

\section{Letras Gregas}

pfg densidade da fase gasosa, $\mathrm{kg} / \mathrm{m} 3$

ps densidade da fase sólida, $\mathrm{kg} / \mathrm{m} 3$

efg fração molar da fase gasosa, $\mathrm{mol} / \mathrm{mol}$

Es fração molar da fase sólida, $\mathrm{mol} / \mathrm{mol}$

$\lambda \quad$ calor latente de vaporização, $\mathrm{kcal} / \mathrm{kg}$

\section{Referências}

[1] Kythe, P. K. Puri, P.; Schaferkotter, M. R., Partial differential equations and Mathematica,. CRC Press, 1997.

[2] Wolfran S., The Mathematica Book, 5 ed., Wolfran Media, 2003.

[3] Silva, J.D.; Lucena, S. "Analysis of the Deterministic Model of a Gas-Solid Fluidized-Bed Gasifier". Journal of Chemical Engineering, 2009.

[4] Silva Teles, A.; Massarani, G., "Condução de Calor em Meios Porosos: Modelo de Duas Fases", Anais do XVIII ENEMP, v.1, pp.175, 1990.

[5] Silva, J.D. "Avaliação da Transferência de Energia e Massa de um Gaseificador de Leito Fluidizado para Geração de Potência", IV Congresso Nacional de Engenharia Mecânica, Conem 2006, Recife. 